\title{
InAs/GaSb superlattices for high-performance infrared detection
}

Rutz, Frank ${ }^{1}$, Rehm, Robert ${ }^{1}$, Schmitz, Johannes ${ }^{1}$, Wauro, Matthias ${ }^{1}$, Niemasz, Jasmin ${ }^{1}$, Masur, JanMichael $^{1}$, Wörl, Andreas ${ }^{1}$, Walther, Martin ${ }^{1}$, Scheibner, Ralf ${ }^{2}$, Wendler, Joachim ${ }^{2}$, and Ziegler, Johann ${ }^{2}$

${ }^{1}$ Fraunhofer-Institute for Applied Solid State Physics IAF, Tullastr. 72, 79108 Freiburg, Germany

${ }^{2}$ AIM Infrarot-Module GmbH, Theresienstr. 2, 74072 Heilbronn, Germany

Photodiodes based on InAs/GaSb short-period superlattices (SLs) have proven their great potential for high-performance infrared (IR) detectors. These heterostructures consist of a periodic series of alternating InAs and GaSb layers with a thickness of only a few atomic monolayers. Hole states are localized in the GaSb layers, whereas the overlapping wave functions of delocalized electronic states in the InAs layers form a miniband for the electrons and enable optical transitions between the holes and the electrons, which are used for the detection of IR radiation. The effective bandgap of this SL structure is lower than the bandgap of the constituting materials InAs and GaSb. The special alignment of the valence and conduction bands in InAs/GaSb is called broken gap type-II band alignment and is depicted in Fig. 1b. Choosing adapted layer thickness values, typically 5-15 monolayers, the cut-off wavelength can be adjusted in the range of $3-30 \mu \mathrm{m}$. The diagram in Fig. 1a shows the influence of the number of atomic monolayers of GaSb and InAs within a superlattice period on the effective bandgap and the detector cut-off wavelength, respectively. The so-called »bandstructure engineering « on the atomic scale became feasible after the development of very precise epitaxial growth techniques, which allow growth of different materials with a few atomic monolayer thickness and well defined interfaces. At Fraunhofer IAF, an molecular beam epitaxy (MBE) machine for simultaneous growth on five 3"-GaSb wafers and one 2" wafer enables the fabrication of InAs/GaSb SLs with excellent homogeneity [1]. Normalized photocurrent spectra of three different InAs/GaSb SL photodiodes with cut-off wavelengths of approximately $9 \mu \mathrm{m}, 5$ $\mu \mathrm{m}$, and $4 \mu \mathrm{m}$ are depicted in Fig. 2. Note, that the specta contain molecular absorption bands, mainly from water vapor and carbon dioxide in the ambient air.

(a)

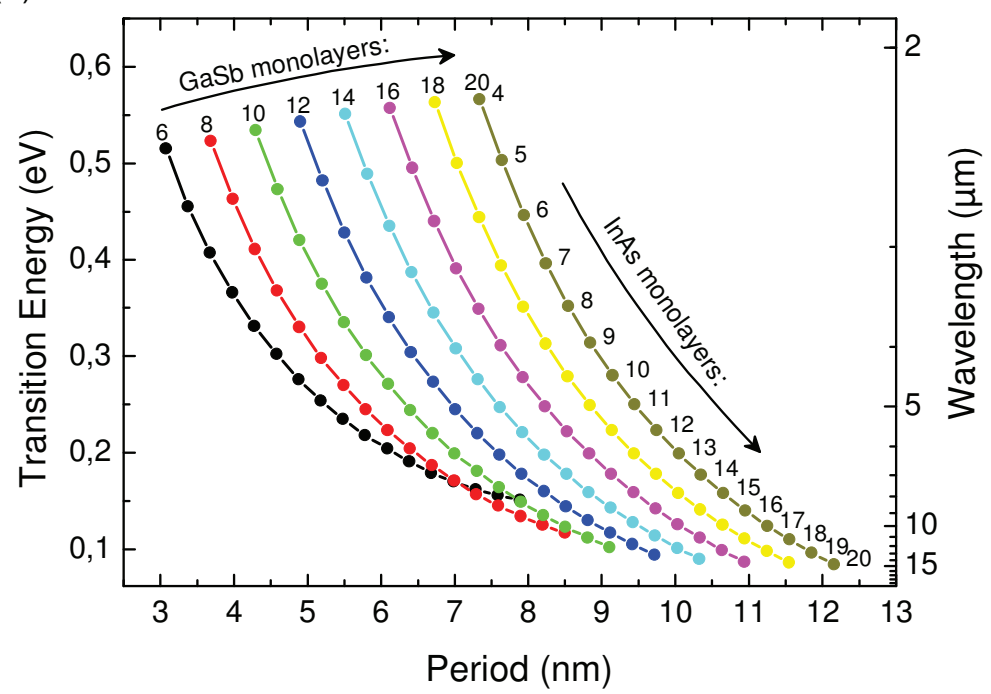

(b)

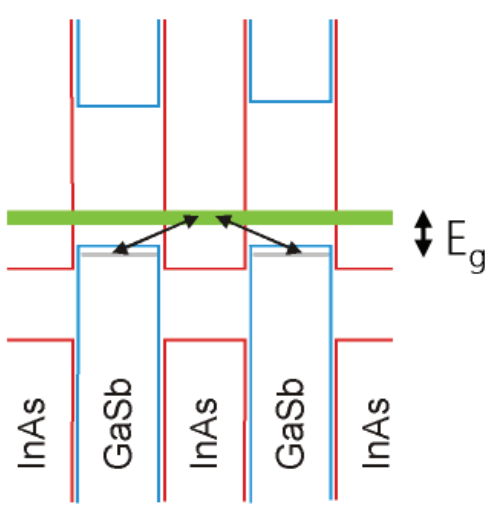

Fig. 1: (a): Transition energy and cut-off wavelength for InAs/GaSb superlattices with different number of monolayers within a single SL period. (b): Band diagram of InAs/GaSb SLs with the effective bandgap $E_{g}$. 


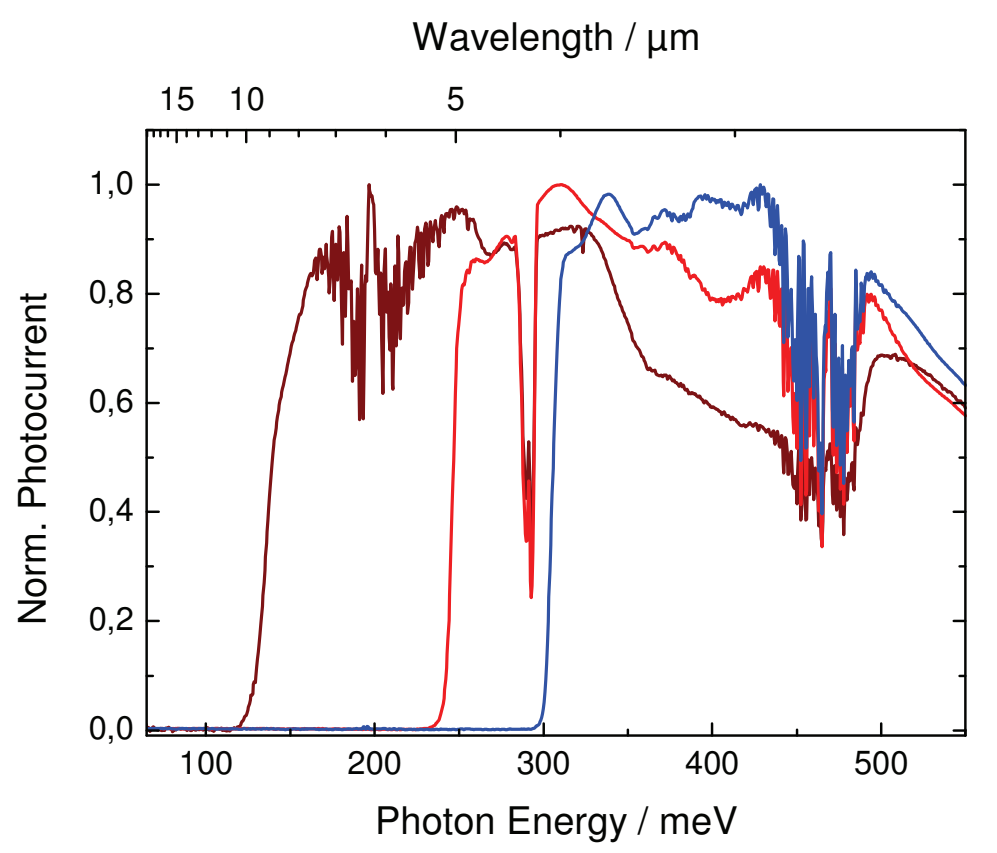

Fig. 2: Normalized photocurrent spectra of $\operatorname{InAs} / \mathrm{GaSb} S L$ detectors with various cut-off wavelengths.

The detector structures are grown on commercial 3" GaSb substrates with low defect density. IR photodiodes are realized by employing an appropriate doping profile along the SL growth axis. The builtin electric field separates photogenerated carriers even without an externally applied bias voltage and thus minimizes the power consumption. The active area is sandwiched between ohmic n- and p-type contacts comprised of a suited combination of doped semiconductor material and a metallization sequence. Since these devices detect the integrated signal of a single wave band they are referred to as monospectral detectors. Fig. 3 shows the vertical structure of a monospectral single detector element.

A well established process technology is used to fabricate focal plane arrays (FPAs) with high pixel yield without large cluster defects and a homogeneous and stable performance. A secondary electron microscopic (SEM) image in Fig. 4a shows a part of a monospectral FPA with a pixel pitch of $24 \mu \mathrm{m}$. The detector chips are hybridized by AIM Infrarot-Module with a custom-designed silicon readout integrated circuit using flip-chip indium solder bump technology. These detector hybrids are mounted into a detector cooler assembly with a $1 \mathrm{~W}$ linear Stirling cooler, which is part of a complete IR imaging camera system.

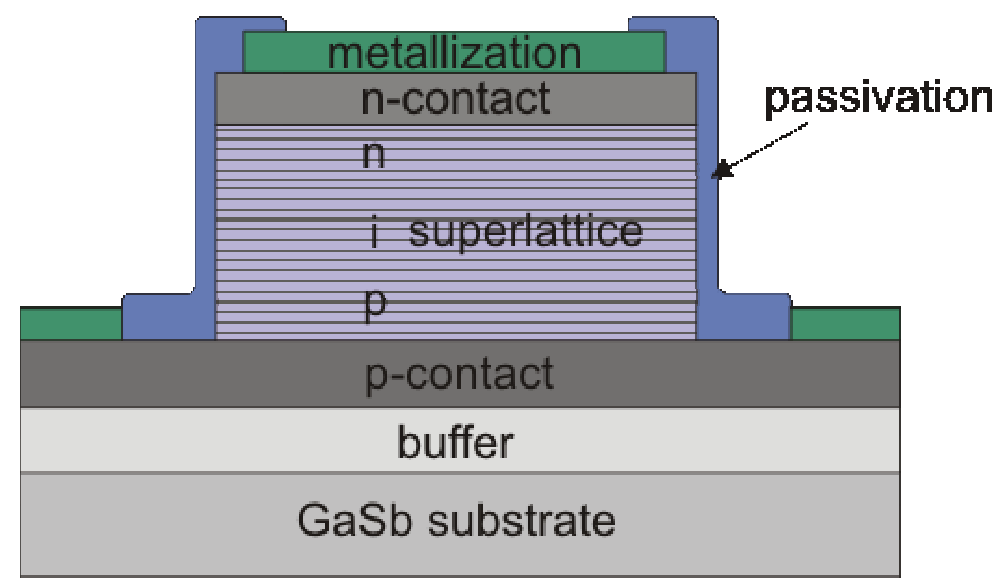

Fig. 3: Scheme of a monospectral single detector element. 


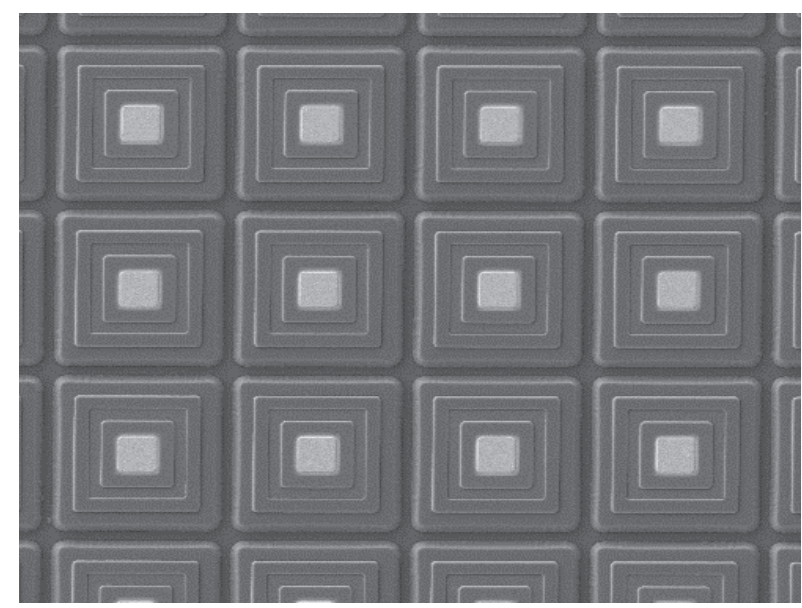

(a)

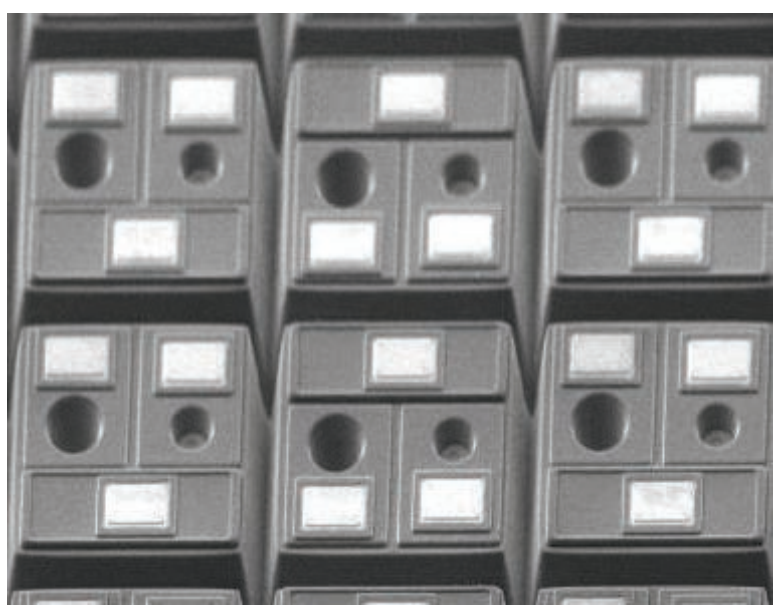

(b)

Fig. 4: SEM images of monospectral (a) and dual-color InAs/GaSb FPA pixels (b) with a pixel pitch of $24 \mu \mathrm{m}$ and $40 \mu \mathrm{m}$, respectively.

The development of InAs/GaSb SL cameras has continuously proceeded. In 2004, the first fully integrated monospectral camera for the mid-wavelength infrared (MWIR, $3-5 \mu \mathrm{m}$ ) band was demonstrated, exhibiting an excellent noise equivalent temperature difference (NETD) below $10 \mathrm{mK}$ with F/2.0 optics [2]. Later, its spatial resolution of $256 \times 256$ pixels was increased to $288 \times 384$ pixels [3]. Arrays with $640 \times 512$ pixels for the MWIR as well as the long-wavelength infrared (LWIR, $8-12 \mu \mathrm{m}$ ) are currently under development.

Beside monospectral MWIR camera systems, Fraunhofer IAF and AIM currently develop the third generation of high-performance IR imaging systems, offering enhanced functionality, i.e. larger spatial resolution and/or multispectral detection. Multispectral IR cameras are able to discern the spectral signature of an object in contrast to monospectral devices, which merely measure the integrated intensity of IR emitters. The transition from monospectral to multispectral IR cameras is comparable with the transition from black-and-white to color photography in the visible. The additional spectral information is of great benefit for image processing. Relative temperature differences can be distinguished very accurately by measuring the integrated (monospectral) intensity if the emissivity of the examined object is known. However, objects with identical temperature but dissimilar emissivity show different intensities in monospectral IR images. For the category of gray bodies, characterized by a wavelength independent emissivity $<1$, bispectral imaging systems can be used to determine the temperature by comparing the signal ratio of both spectral channels. Furthermore, the wavelength dependent transmission and absorption properties can be utilized to distinguish different solid materials, liquids, or gases.

The determination of the intensity distribution in various spectral ranges (»colors «) is usually realized by implementing filters. The main disadvantage of this method is that only one color is observed at a time, i.e. simultaneous image acquisition in various wavelength ranges on the same pixel is not possible. Hence, the development of high-resolution thermal imagers for simultaneous detection of IR radiation in two spectral ranges on a single detector chip is beneficial. For a bispectal detector, this can be achieved by stacking two monospectal SL layers on top of each other. The first stack absorbs IR photons with a higher energy (»blue color «), whereas the second stack is sensible for photons with a lower energy (»red color «) [4]. In each stack, a p-i-n photodiode is realized by appropriate p- and n-doping. Both p-i-n diodes are placed back-to-back with a common p-type mass contact enabling the synchronous read-out of both diodes. The layer structure of the dual-color detector exhibit cut-off wavelengths around $4 \mu \mathrm{m}$ and $5 \mu \mathrm{m}$, respectively (cf. Fig. 2).

Dual-color FPAs are fabricated with $288 \times 384$ pixels at $40 \mu \mathrm{m}$ pitch. FPA processing includes the drychemical etching of contact via holes to the lower contact layers, followed by trench etching for pixel isolation. Each pixel features three electrical contacts for simultaneous detection of both colors. A part of a dual-color FPA is shown in the SEM image in Fig. 4b. Recently, NETD values of $18 \mathrm{mK}$ have been measured for the short wavelength detector and $10 \mathrm{mK}$ for the long wavelength detector with F/2.0 optics 
and $1.5 \mathrm{~ms}$ integration time. NETD histograms and sample images of the individual dual-color channels are depicted in Fig. 5 and 6, respectively.
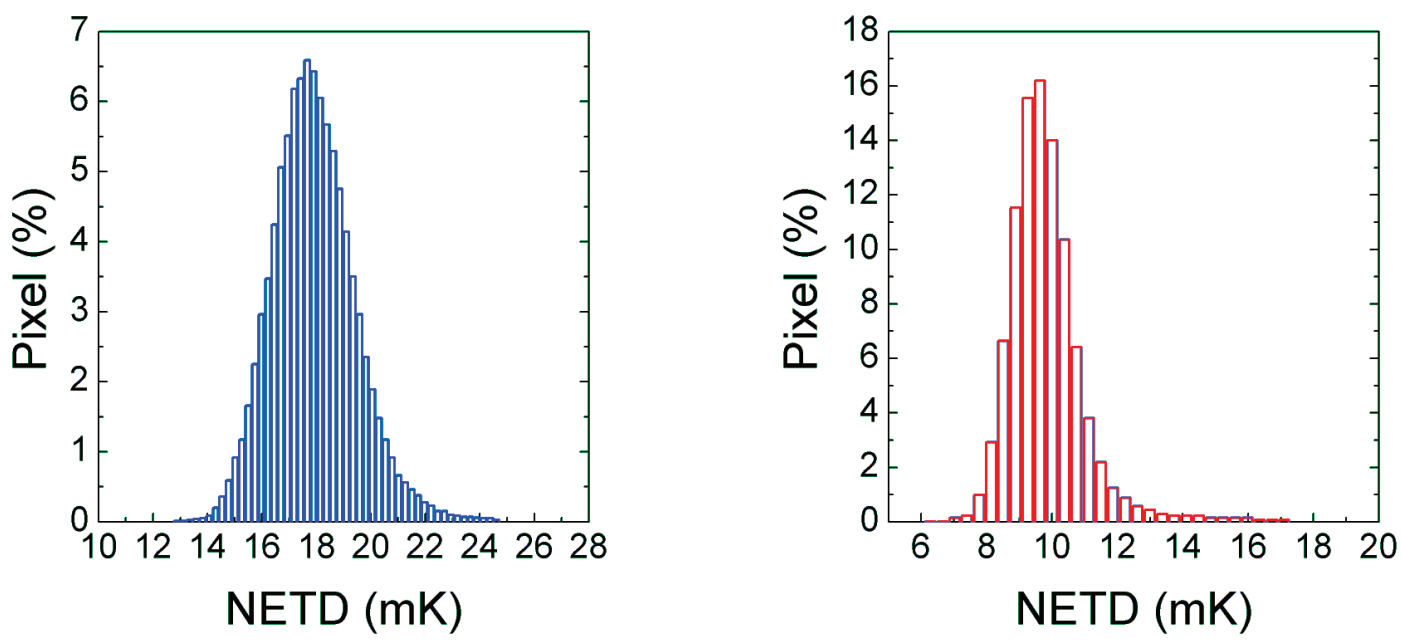

Fig. 5: NETD histograms of the »blue « channel (a) and the »red« channel (b) of a $288 \times 384$ MWIR/MWIR dual-color InAs/GaSb SL camera system.

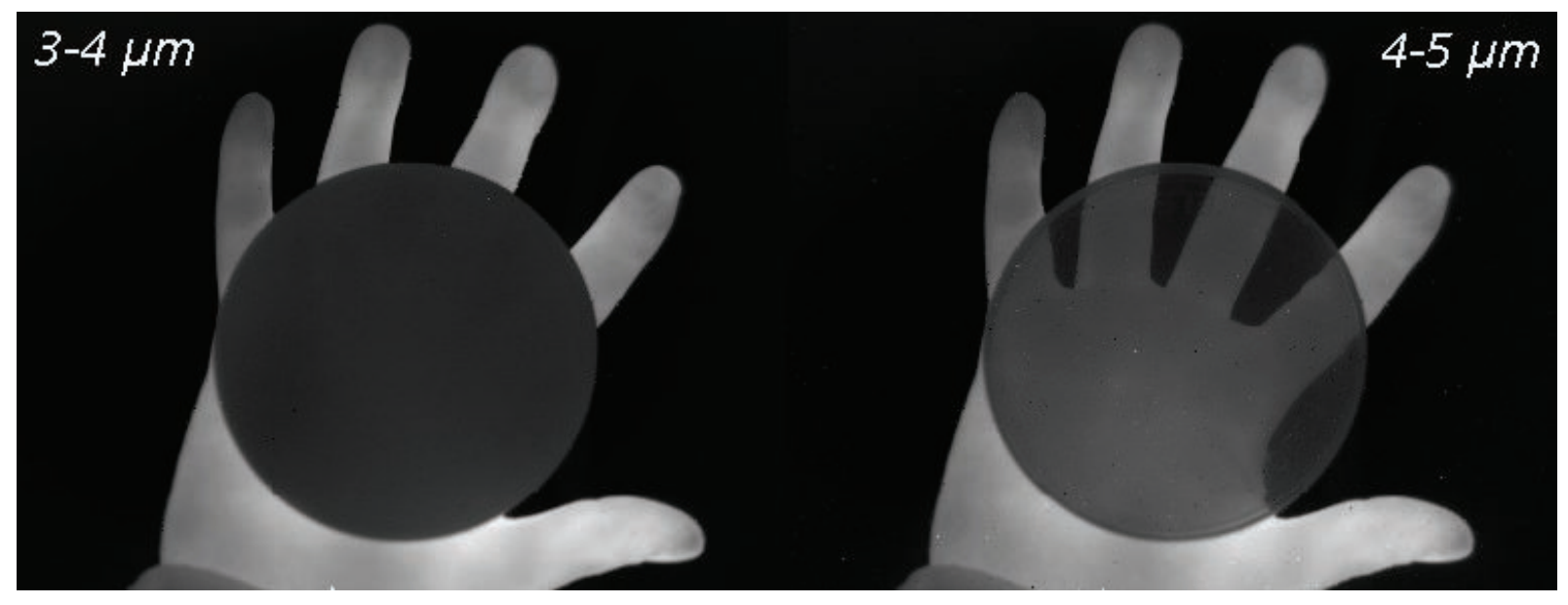

Fig. 6: Dual-color infrared image showing a hand holding a plastic lid with varying transparency in both bands.

Such dual-color detectors are ideally suited for remote detection of carbon dioxide due to the strong $\mathrm{CO}_{2}$ line around $4.3 \mu \mathrm{m}$ (cf. Fig. 2). Since IR emission form $\mathrm{CO}_{2}$ occurs in the »red « but not in the »blue channel «, the differences between these two color channels is a very sensitive indicator for $\mathrm{CO}_{2}$. A bispectral infrared image of an industrial site taken with such a dual-color InAs/GaSb SL camera is presented in Fig. 7. The images of the two MWIR color channels $3-4 \mu \mathrm{m}$ and $4-5 \mu \mathrm{m}$ are superimposed by the complementary colors cyan and red, respectively. The red signatures in Fig. 7 clearly reveal IR emissions from hot $\mathrm{CO}_{2}$ in the scene, whereas scatterings especially from water vapor, e.g. from steam exhausts or in clouds, appear bluish due to the frequency dependency of the Rayleigh scattering coefficient. Via a spatially and temporally coherent detection of two different wavelength ranges in the MWIR, the dual-color SL imagers enable fast and highly sensitive remote detection of carbon dioxide. This kind of dual-color detectors is integrated into missile warning systems in the first procurement program exploiting a third generation IR imager. Further applications range from remote detection of gas leakages to the investigation of the $\mathrm{CO}_{2}$ gas exchange between the ocean and the atmosphere and its influence on climate change. Basically, by selecting different cut-off wavelengths for 
the two dual-color channels, the detection of other gases, e.g. methane, carbon monoxide, etc., is also possible.

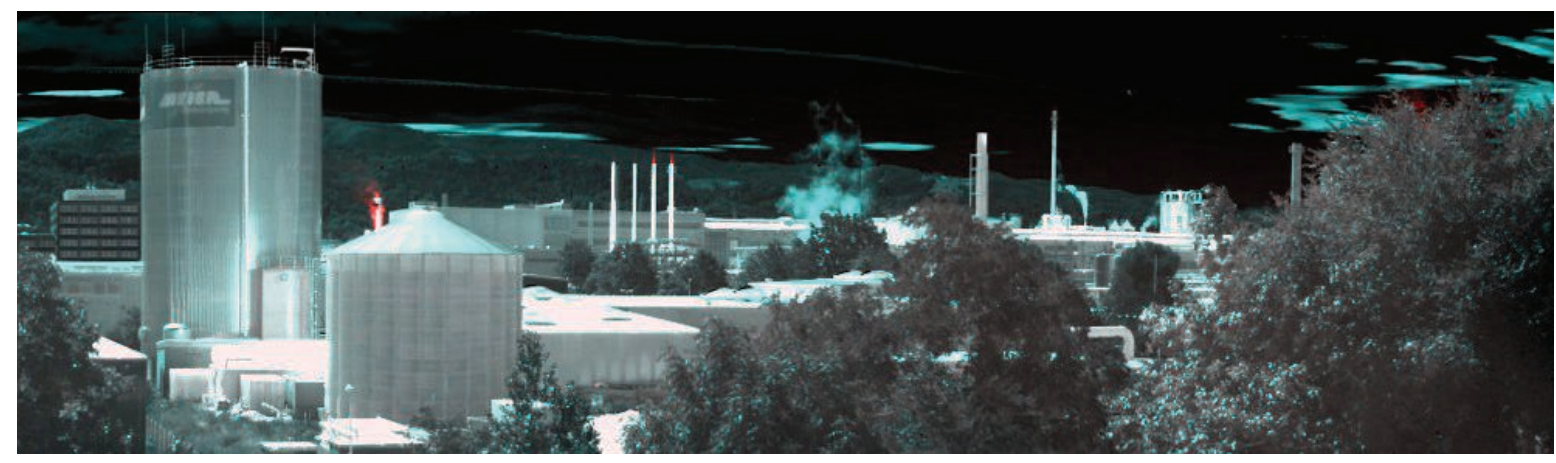

Fig. 7: Bispectral infrared image of an industrial site taken with a $384 \times 288$ dual-color $\ln A s / G a S b$ superlattice camera. The two color channels $3-4 \mu \mathrm{m}$ and $4-5 \mu \mathrm{m}$ are represented by the complementary colors cyan and red, respectively.

\section{References}

[1] Rutz, F., Rehm, R., Schmitz, J., Fleissner, J., Walther, M., Scheibner, R., and Ziegler, J., "InAs/GaSb superlattice focal plane array infrared detectors: manufacturing aspects", Proc. SPIE 7298, 72981R (2009).

[2] Rehm, R., Walther, M., Schmitz, J., Fleißner, J., Fuchs, F., Cabanski, W., and Ziegler, J., "InAs/(Galn)Sb short-period superlattices for focal plane arrays", Proc. SPIE 5783, 123 (2005).

[3] Rehm, R., Walther, M., Schmitz, J., Fleißner, J., Ziegler, J., Cabanski, W., and Breiter, R., "Second and third generation thermal imagers based on type-II superlattice photodiodes", Proc. SPIE 6294, 629404 (2006).

[4] Rehm, R., Walther, M., Schmitz, J., Rutz, F. , Wörl, A., Scheibner, R., Ziegler, J., "Type-II Superlattices - The Fraunhofer Perspective", Proc. SPIE 7660, 76601G (2010). 